\title{
Important Role of Translational Science in Rare Disease Innovation, Discovery, and Drug Development
}

\author{
Anne R. Pariser, $M D^{7}$ and William A. Gahl, MD, $P h D^{2}$ \\ 'Office of New Drugs, Rare Diseases Program, Center for Drug Evaluation and Research, Food and Drug Administration, Silver Spring, MD, \\ USA ${ }^{2} \mathrm{NIH}$ Undiagnosed Diseases Program, Office of the Director and National Human Genome Research Institute, National Institutes of \\ Health, Bethesda, MD, USA.
}

Rare diseases play a leading role in innovation and the advancement of medical and pharmaceutical science. Most rare diseases are genetic disorders or atypical manifestations of infectious, immunologic, or oncologic diseases; they all provide opportunities to study extremes of human pathology and provide insight into both normal and aberrant physiology. Recently, drug development has become increasingly focused on classifying diseases largely on genetic grounds; this has allowed the identification of molecularly defined targets and the development of targeted therapies. Clinical trials are now focusing on progressively smaller subgroups within both common and rare disease populations, often based on genetic tests or biomarkers. Drug developers, researchers, and regulatory agencies face a variety of challenges throughout the life cycle of drug research and development for rare diseases. These include the small numbers of patients available for study, lack of knowledge of the disease's natural history, incomplete understanding of the basic mechanisms causing the disorder, and variability in disease severity, expression, and course. Traditional approaches to rare disease clinical research have not kept pace with advances in basic science, and increased attention to translational science is needed to address these challenges, especially diagnostic testing, registries, and novel trial designs.

KEY WORDS: rare disease; translational medical research; diagnosis; registries.

J Gen Intern Med 29(Suppl 3):S804-7

DOI: $10.1007 / \mathrm{s} 11606-014-2881-2$

(c) Society of General Internal Medicine 2014

$\mathrm{W}$ hile considerable progress has been made in advancing our understanding of the etiology of many rare diseases, basic scientific advances have not always resulted in direct benefits to patients. Rare diseases (also known as orphan diseases) are officially defined as diseases or conditions affecting fewer than 200,000 people in the U.S. ${ }^{1}$ There are an estimated 7,000 different rare diseases,

Published online July 17, 2014 but only 250 to 300 of them are treatable with drugs approved in the U.S. ${ }^{2}$ One of the greatest challenges in moving rare disease drug development forward is the difficulty of conducting clinical programs in small populations. Translational science, which provides the information upon which rational clinical development programs are built, has often lagged behind the rapid pace of basic scientific innovation. Therefore, this area of rare disease research deserves additional attention if benefits to patients are to be realized. If basic science can be said to encompass the development and establishment of information to aid understanding, then translational science addresses practical, cross-disciplinary applications to directly help people and bridge the gap between bench and bedside. Translational science is broad enough to encompass many different disciplines, including drug discovery, assay and diagnostic development, mechanistic and proof-of-concept research, and the study of the natural history of diseases, all of which play important roles in moving potential treatments from discovery through to clinical investigations.

Rare diseases have long had a leading role in innovation and the advancement of medical science, largely because they reveal mechanisms that are not apparent when systems and pathways are functioning normally (e.g., absence or deficiency of an endogenous enzyme). Rare diseases affect all therapeutic areas and patients across all demographic subgroups. Most rare diseases are genetic disorders that result in alterations in biochemical or physiologic pathways, but many are acquired or involve atypical manifestations of more common diseases. Examples include infectious agents (e.g., multi-drug resistant tuberculosis ${ }^{3}$ ), malignancies (e.g., mutation-defined cancer subsets, such as alkaline lymphoma kinase-positive non-small cell lung cancer ${ }^{4}$ ), neurological conditions with an autoimmune basis (e.g., GAD-65 antibody-positive stiff-man syndrome or neuromyelitis optica), or rheumatologic disease (e.g., ANCAassociated vasculitides ${ }^{5}$ ). As such, rare diseases present opportunities to learn from the extremes of human pathology, providing a window into both normal and aberrant physiology and helping to unravel the intricacies of human biology for the benefit of patients with both common and rare diseases. In recent years, some of the most notable advances in medical and pharmaceutical science have focused on progressively smaller subgroups within disease populations, often based on genetic 
tests or biomarkers. This has led to the classification of some common diseases as rare (e.g., mutation-defined subsets of cancers), and to classifying rare diseases into smaller and smaller subpopulations (e.g., mutation-defined subsets of cystic fibrosis ${ }^{6}$ ).

Such subdivision of diseases on genetic grounds has allowed the identification of molecularly defined targets, development of targeted therapies, and selection of patients for treatment or for inclusion into pivotal clinical trials based on genetic tests, biomarkers, or susceptibility of tumors or microorganisms. Between 2010 and 2012, targeted therapies, such as for BRAF V600 mutation melanoma, ${ }^{7,8}$ represented about $25 \%$ of all novel drug approvals and half of novel rare disease drug approvals at the U.S. Food and Drug Administration (FDA)'s Center for Drug Evaluation and Research (CDER), up from only about $10 \%$ of novel drug approvals from 2000 to $2002 .{ }^{9}$ While targeted therapies offer interventions focused on the underlying cause of disease, with potentially increased efficacy and safety, they also present challenges, especially in the evaluation of novel agents in small clinical trials.

\section{TRANSLATIONAL SCIENCE}

In order to transform rare disease research and move drug development efficiently forward, a combination of translational scientific approaches will be needed. These include the use of improved diagnostic and patient-selection methods, innovative and flexible clinical trial designs and statistical approaches, and novel techniques for describing, monitoring and interpreting disease outcomes and effects of intervention in small and/or enriched populations (for example, patients selected for inclusion in a clinical trial or for treatment based on a biomarker or specific genetic mutation, such as BRAF V600 mutation positive melanoma ${ }^{7,8}$.

\section{Diagnosis}

Diagnosis has been a sadly neglected area of rare disease research. Patients with rare diseases frequently describe a long and difficult journey toward obtaining a diagnosis, fraught with frustration and desperation. They also note the need for physicians to be better trained in rare diseases in order to speed diagnosis. ${ }^{10}$ Fortunately, advanced technologies such as single nucleotide polymorphism (SNP) arrays, exome sequencing, and genome sequencing are facilitating the diagnosis of both rare and new diseases. ${ }^{11}$ In addition, scientists, physicians, and the general public now recognize the critical importance of diagnosis in any attempt to offer therapy. ${ }^{12}$ This provides hope for a sustained campaign to identify rare disease patients utilizing the Human Genome Project, which continues to help identify subpopulations for targeted cancer and metabolic disease therapies. For example, a boy with uncontrolled seizures was diagnosed with specific GRIN2A mutations that caused enhanced agonist potency for the NMDA receptor subunit GluN2A. ${ }^{13}$ The in vitro demonstration of inhibition of this type of hyperexcitability using memantine allowed for targeted therapy with this child, with the potential to benefit other individuals with similar GRIN2A mutations. ${ }^{14}$

One example of current interest in diagnosis is the success of the National Institutes of Health (NIH) Undiagnosed Diseases Program (UDP). ${ }^{15-17}$ Established in 2008, the UDP has investigated 700 undiagnosed individuals during one-week inpatient admissions at the NIH Clinical Center, where extensive phenotyping and critical assessments are performed. More than 60 diseases of very low prevalence have been diagnosed, and an equal number of potential new disease genes have been identified; most of them await verification through functional testing. The NIH Common Fund has allocated $\$ 145 \mathrm{M}$ over fiscal years 2013-2020 to support five to seven extramural UDPs at medical centers throughout the country, a data coordinating center, a training program in exome sequence analysis, and gene function studies. Additional undiagnosed disease centers have been established using only institutional funds. The sharing of de-identified phenotypes and exome sequence variants should yield new rare disease diagnoses. The concept of UDPs has elicited worldwide interest, and plans are underway to establish an international consortium to synergize the diagnostic efforts of UDP centers around the world.

\section{Registries}

Registries are often the first step toward research into a rare disease. Registries encompass diverse types of data collection efforts, ranging from databases for patient communication and prevalence estimates, to broad-based collections of safety information and assessments of outcomes for rare disease therapeutics. Registries permit the development of non-traditional data collection methods, such as from social media and data collected for other purposes (e.g., medical, billing or pharmacy records), and can support extended follow-up of geographically dispersed patients. Registries have also led to non-traditional partnering, such as between advocacy groups and industry, and have enhanced the role of advocates in research and patient care. One example is the Global Rare Diseases Patient Registry and Data Repository (GRDR), a pilot project launched by the Office of Rare Diseases Research at NIH. ${ }^{18}$ The GRDR was established to develop a standardized data repository across many rare diseases that could support analyses in a variety of research projects, such as clinical trials and drug development. Other examples include disease-specific registries, such as the Cystic Fibrosis Foundation (CFF) patient registry, which was founded about 40 years ago and has helped to establish disease care practices, improve 
patient outcomes, and guide the CFF scientific agenda, among other achievements. ${ }^{19}$

\section{Clinical Trial Design}

Many diverse clinical trial designs have been used to good effect for rare diseases, ${ }^{20}$ and researchers in the field, drug developers, and regulatory agencies have shown great flexibility in enhancing and expanding upon traditional methods. Experts have published algorithms and descriptions of alternative designs for small populations and specific situations, such as Bayesian methods and adaptive designs; a full description of these methods is beyond the scope of this commentary. ${ }^{21,22}$ Two examples of trial designs that may be of particular interest for biomarkerdefined rare diseases are basket and multi-arm trials. Basket trials are designed to test the effects of a targeted agent against a specific molecular mutation regardless of histologic context. ${ }^{21}$ Histologically defined tumors in cancer are typically heterogeneous and consist of many smaller molecularly defined subsets. When the anti-tumor activity of a drug is expected to affect a specific molecular aberration, basket trials may include patients with different histologically defined tumors but common molecular mutations. Multiple-arm trials use one control arm (e.g., placebo) with which more than one experimental agent can be compared. ${ }^{22}$ Both basket and multiple-arm designs allow for the efficient and parsimonious use of small populations, but they also require unprecedented levels of cooperation, such as between developers of competing drugs, and close collaboration among disease experts in different therapeutic areas.

\section{FUTURE DIRECTIONS}

The year 2013 marked the 30th anniversary of the Orphan Drug Act. This landmark legislation spurred tremendous growth in orphan drug development and approvals, largely through financial incentives during the clinical phases of development and in the post-approval period. However, in addition to financial considerations, the rare disease community (including academia, the drug industry, patients and advocates, and regulatory agencies) faces a variety of impediments to movement through the life cycle of orphan drug research and development. These include lack of knowledge of the natural history and the basic mechanisms of the disease, variability in disease severity, expression, and - of course - a paucity of patients for clinical trials. Traditional paradigms for rare disease clinical research have not kept pace with pre-clinical advances. In fact, our entire approach to rare disease research and development-from basic science to clinical investigation-warrants re-think- ing. While many elements of translational science have already been described for common diseases, such as disease natural history and diagnostic strategies, most rare diseases lack this existing infrastructure; translational science may need to be developed in advance of or in parallel with traditional drug development approaches. This edition of the Journal of General Internal Medicine describes several novel and creative approaches used by research-investigators to address some of the gaps identified in rare disease research.

While the number of people affected with an individual rare disease is small, rare diseases collectively afflict 25-30 million Americans, and advances in diagnostics are adding to these numbers daily. Resource allocation remains grossly inadequate for the magnitude of the problem. For example, cancers and HIV, which together afflict approximately 17 million Americans, receive far greater public resource allocation; large-scale, sustained public health prioritizations have produced dramatic improvements in the lives of patients with HIV and cancer. A commensurate commitment of public funding for rare diseases could lead to similar advances for rare disease patients, most of whom currently have no therapies available for their treatment. This is especially urgent as advances in genetics and pharmaceutical science move us closer to personalized medicine, the ultimate goal of every rare disease patient and caregiver.

Acknowledgements: This work was supported in part by the Intramural Research Program of the National Human Genome Research Institute.

Conflict of Interest: The authors declare that they do not have a conflict of interest.

Disclaimer: The opinions presented herein are those of the authors and do not represent the views or policies of the U.S. Food and Drug Administration or the National Institutes of Health.

Corresponding Author: Anne R. Pariser, MD; Office of New Drugs, Rare Diseases Program, Center for Drug Evaluation and ResearchFood and Drug Administration, WO22-6471, 10903 New Hampshire Ave, Silver Spring, MD 20993, USA (e-mail: Anne.pariser@fda.hhs.gov).

\section{REFERENCES}

1. Orphan Drug Act, Pub. L. 97-414. 96 Stat. 2049 (1983). Amended in 1984 by Pub. L. $98-551$ to add a numeric prevalence threshold to the definition of rare diseases.

2. U.S. Food and Drug Administration (FDA). Search orphan drug designations and approvals. Available at: http://www.accessdata.fda.gov/ scripts/opdlisting/oopd/. Accessed February 5, 2014.

3. Clark TG, Mallard K, Coll F, et al. Elucidating emergence and transmission of multidrug-resistant tuberculosis in treatment experienced patients by whole genome sequencing. PLoS One. 2013;8:e83012. doi:10.1371/journal.pone.0083012.

4. Shaw AT, Kim D, Nakagawa $\mathbf{K}$, et al. Crizotinib versus chemotherapy in advanced ALK-positive lung cancer. N Engl J Med. 2013;368:2385-94. 
5. Craven A, Robson J, Ponte C, et al. ACR/EULAR-endorsed study to develop Diagnostic and Classification Criteria for Vasculitis (DCVAS). Clin Exp Nephrol. 2013;17:619-21.

6. Ramsey BW, Davies J, McElvaney NG, et al. A CFTR potentiator in patients with cystic fibrosis and the G551D mutation. N Engl J Med. 2011;365: 1663-72.

7. Sosman JA, Kim KB, Schuchter L, et al. Survival in BRAF V600mutant advanced melanoma treated with vemurafenib. N Engl J Med. 2012;366:707-14.

8. Flaherty KT, Infante JR, Daud A, et al. Combined BRAF and MEK inhibition in melanoma with BRAF V600 mutations. N Engl J Med. 2012;367:1694-703.

9. U.S. FDA. Drugs@FDA. Available at: http://www.accessdata.fda.gov/ scripts/cder/drugsatfda/index.cfm. Accessed March 19, 2014.

10. Engel PA, Bagal S, Broback M, Boice N. Physician and patient perceptions regarding physician training in rare diseases: The need for stronger educational initiatives for physicians. J Rare Dis. 2013;1:115.

11. Yang Y, Muzny DM, Reid JG, et al. Clinical whole-exome sequencing for the diagnosis of Mendelian disorders. N Engl J Med. 2013;369:150211.

12. Gahl WA. The battlefield of rare diseases: where uncommon insights are common. Sci Transl Med. 2012;4:1-3.

13. Yuan $\mathbf{H}$, Hansen $\mathbf{K B}$, Zhang $\mathbf{J}$, et al. Functional analysis of a de novo GRIN2A missense mutation associated with early-onset epileptic en cephalopathy. Nat Commun. 2014;5:3251. doi:10.1038/ncomms4251.
14. Pierson TM, Yuan H, Fuentes Fajardo $\mathbf{K}$, et al. De novo GRIN2A mutation results in early-onset epileptic encephalopathy; Personalized therapy with memantine. Ann Clin Transl Neurol. 2014, in press.

15. Gahl WA, Tifft CJ. The NIH undiagnosed diseases program: lessons learned. JAMA. 2011;305:1904-5.

16. Gahl WA, Markello TC, Toro C, et al. The National Institutes of Health undiagnosed diseases program: insights into rare diseases. Genet Med. 2012;14:51-9.

17. Gahl WA, Boerkoel CF, Boehm M. The NIH undiagnosed diseases program: bonding scientists and clinicians. Dis Model Mechanobiol. 2012;5:3-5.

18. NIH, National Center for Advancing Translational Sciences, Office of Rare Diseases Research. Global Rare Disease Patient Registry and Data Repository (GRDR). Available at: https://rarediseases.info.nih.gov/research/pages/43/global-rare-disease-patient-registry-and-data-repository. Accessed February 5, 2014.

19. Cystic Fibrosis Foundation. Patient Registry Report. Available at: http:// www.cff.org/livingwithcf/qualityimprovement/patientregistryreport/ Accessed February 5, 2014.

20. Sasinowski FJ. Quantum of effectiveness evidence in FDA's approval of orphan drugs. Drug Inf J. 2012;46:238-63.

21. Sleijfer S, Bogaerts J, Siu LL. Designing transformative clinical trials in the cancer genome era. J Clin Oncol. 2013;31:1834-41.

22. Korn EL, McShane LM, Freidlin B. Statistical challenges in the evaluation of treatments for small patients populations. Sci Transl Med. 2013;5:1-14. 